\title{
Hybrid Homomorphic Encryption Method for Protecting the Privacy of Banking Data in the Cloud
}

\author{
Maha Tebaa ${ }^{1}$, Karim Zkik ${ }^{2}$ and Said El Hajji ${ }^{3}$ \\ 1, 2, ${ }^{3}$ University of Mohammed-V, Faculty of Sciences - Rabat, Morocco, \\ Laboratory of Mathematics, Computing and Applications \\ ${ }^{1}$ maha.tebaa@gmail.com, ${ }^{2}$ karim.zkik@gmail.com, ${ }^{3}$ elhajji@fsr.ac.ma
}

\begin{abstract}
The time and cost saving for banks when implementing cloud computing strategies are staggering. However, it is important to consider the security and protection of data when it comes to the widespread adoption of cloud. Fully homomorphic encryption is currently still undergoing experimentations. One of its limitation is the time required to encrypt and decrypt the sensitive data, as the traditional encryption systems showed a level of resistance and considerable maturity that can be rehabilitated or hybridized for application in the field of sensitive data protection hosted in the Cloud.

In this paper we will propose hybrid homomorphic system that will be applied to the banking data to perform operations on encrypted data without decrypting, based on the encrypting, the decryption and the operation treatment time on the ciphertext which were obtained by simulating an addition and multiplication homomorphic cryptosystem and comparing it with DGHV that is somewhat homomorphic; then we will choose our model which is most suitable for banking application.
\end{abstract}

Keywords: Homomorphic Encryption, Paillier, RSA, Cloud Computing, bank data

\section{Introduction}

Companies do not longer refute the need of storing its sensitive data on the cloud, which they already do using traditional encryption systems. The question here is how to process this data without decrypting it in order to respect the company's privacy. Hence the idea of using homomorphic encryption for banking data which is a new approach that can help banks take their data management to the highest level: the cloud [1].

Cloud Computing is not only a new seductive marketing trend, but it is an economic reality to access many services online. This comprehensive access offer to computing resources allows evolutionarily companies to focus on their core competencies by exempting them from their servers' management. The major question that keeps many companies reluctant regarding this new storing technology, including banks, is the security of their sensitive data hosted on the Cloud.

Cloud computing may offer several advantages in terms of cost reduction and scalability but raises new security issues regarding sensitive data.

Following this, and in order to perform operations on encrypted banking data stored in the cloud without decrypting them, we will opt for the homomorphic encryption $[2,3]$. This latter consists in encrypting the client's identity with a multiplicative homomorphic encryption which corresponds to the "logical and" used for keyword searching into an encrypted database. Add to this, the data concerning a client's deposits of all his or her accounts is encrypted by an additive homomorphic encryption; aiming to compute the total amount of the client's deposits by the cloud server and deciphering the result at the level of client's private bank. In both cases, the result is the same, as if the calculations were made using raw data. 
In Section 2, we will talk about the lack of Cloud adoption by banks. In Section 3, we will define Homomorphic Encryption and we will illustrate some examples of existing Homomorphic cryptosystems. In Section 4, the focus will be on our simulation and its analysis, in Section 5; we will present the scheme of our banking application with some examples of calculations on banking accounts of customers. The conclusion and perspectives are dealt with in Section 5.

\section{Banking in the Cloud}

The lack of cloud services usage, not only by banks but also by all the companies in all the fields, creates a strong incentive for the researches and cloud providers to adapt their solutions to the security required by banks, encouraging them to migrate and to outsource their computations to the cloud; allowing them to focus only on their core competencies.

The migration to the cloud will lead to a control loss over the customer bank data (account numbers and sum of deposits, loans allocations...).

There are many compelling reasons to migrate applications and data to a private or a public cloud: scalability, agility, cost savings and so on and so forth. However, those benefits come along with a new risk regarding the security of critical business data; a risk that has to be managed by any organization whose data migrated to the cloud using a robust solution for data encryption and encryption key management. This risk is illustrated through the USA PATRIOT Act [4]; an act that makes it possible for competitors and governments to access data through cloud providers without the consent of the data owner. Even storing data in foreign jurisdictions does not provide a sufficient guaranty that customers' data is out of reach of disclosure requirements.

To solve this problem, some security vendors require the installation of a physical key management server in the data center as a prerequisite. Other security providers require you to "trust" them and use their key management service. This approach violates the principle and the respect of the requirements keeping the keys under your own control.

The banking sector is renowned for the volume and speed of data it produces, transports and stores. Given the recent financial crisis and subsequent regulatory pressures, the cost of computers has grown exponentially not only for the banking organization as a whole, but even at the level of the division.

Nowadays, Banks have to cut their IT costs, but it should not be done at the stake of compromising their data security and integrity. The need, therefore, to explore the unconventional and innovative options reduced IT costs while maintaining data integrity. However, with limited resources and even limited budgets, a methodology for traditional IT implementation is neither effective nor desired [5].

The Cloud Computing [6], as a technology option for data and business processes, took credible foothold in recent times in several industry sectors including financial services. This paper details the "must knows" to implement an efficient and secure information management framework in a cloud environment. We will, first, explore the various delivery and deployment of cloud models available to a bank.

The security must be your cloud provider's top priority. As matter of fact, the highest portion of Amazon Web Services (AWS) investments is dedicated to increase this level of security is based on a model of shared responsibility: AWS [7] ensures the security of the lower layers (physical buildings, networks, virtualization...), and you are responsible for the security of your applications, OS and data on which you have total control (e.g., administrator access to VM).

This means that you apply the same rules of security on your applications in the cloud infrastructure. 


\section{Homomorphic Cryptosystems}

Cloud Encryption is the only solution that offers the convenience of encryption and key management in virtualized environments, without giving up data confidentiality.

Security requirements for data and algorithms have become stricter throughout the latest years. Due to the large growth of the technology, a wide variety of attacks on digital assets and technical devices are activated. For storage and safe data reading, there are several possibilities such as data encryption. The problem becomes more complex when asking to perform computation on encrypted data. This is where the homomorphic cryptographic systems can be used.

The partial homomorphic encryption allows some operations on encrypted data without decrypting it, examples: RSA, Paillier.

\subsection{Multiplicative Homomorphic Encryption}

The multiplicative homomorphic encryption
$D_{k}\left(E_{k}(n) \times E_{k}(m)\right)=n \times m$ OU $E_{k}(x \otimes y)=E_{k}(x) \otimes E_{k}(y)$

Example of Multiplicative Homomorphic Encryption (RSA) [8]:

\section{RSA : Multiplicative homomorphic encryption}

Let $n=p q$, such that $\mathrm{p}$ and $\mathrm{q}$ are relatively prime, $\mathrm{a}$ and $\mathrm{b}$ are selected such that $a b \equiv 1(\bmod \Phi(n))$.

$\mathrm{n}$ and $\mathrm{b}$ are public; $\mathrm{p}, \mathrm{q}$ and are private.

$$
\begin{aligned}
& E_{k}(x)=x^{b} \bmod n \\
& D_{k}(y)=y^{a} \bmod n
\end{aligned}
$$

$\mathrm{E}_{\mathrm{k}}\left(\mathrm{x}_{1}\right) \times \mathrm{E}_{\mathrm{k}}\left(\mathrm{x}_{2}\right)=\mathrm{x}_{1}{ }^{\mathrm{b}} \mathrm{x}_{2}{ }^{\mathrm{b}} \bmod \mathrm{n}=\left(\mathrm{x}_{1} \mathrm{x}_{2}\right)^{\mathrm{b}} \bmod \mathrm{n}=\mathrm{E}_{\mathrm{k}}\left(\mathrm{x}_{1} \mathrm{x}_{2}\right)$

\subsection{Additive Homomorphic Encryption}

$$
\begin{aligned}
& \text { The additive homomorphic encryption } \\
& D_{L}\left(E_{L}(n) \times E_{L}(m)\right)=n+m \text { OU } E_{k}(x \oplus y)=E_{k}(x) \otimes E_{k}(y)
\end{aligned}
$$

Example of Additive Homomorphic Encryption (Paillier) [9]:

\section{Paillier : Additive homomorphic encryption}

Let $\mathrm{p}$ and $\mathrm{q}$ be two large prime numbers, et $n=p q$. $\lambda$ denotes the Carmichael function as, $\lambda(n)=\operatorname{gcm}(p-1, q-1)$.

Let $g \in \mathbb{Z}_{n}^{*}$ a random as $L\left(g^{\lambda} \bmod n^{2}\right)$ is invertible modulo $\mathrm{n}$ (où $L(u)=\frac{u-1}{u}$ ). $\mathrm{n}$ and $\mathrm{g}$ are public; $\mathrm{p}$ et $\mathrm{q}$ ( or $\lambda$ ) are private.

$$
\begin{gathered}
E_{k}(x, r)=g^{x} r^{n} \bmod n^{2} \\
D_{k}(y)=\frac{L\left(y^{\lambda} \bmod n^{2}\right)}{L\left(g^{\lambda} \bmod n^{2}\right)} \bmod n
\end{gathered}
$$

$\mathrm{E}_{\mathrm{k}}\left(\mathrm{x}_{1}, \mathrm{r}_{1}\right) \times \mathrm{E}_{\mathrm{k}}\left(\mathrm{x}_{2}, \mathrm{r}_{2}\right)=\mathrm{g}^{\mathrm{x} 1+\mathrm{x} 2}\left(\mathrm{r}_{1} \mathrm{r}_{2}\right) \bmod \mathrm{n}^{2}=\mathrm{E}_{\mathrm{k}}\left(\mathrm{x}_{1}+\mathrm{x}_{2}, \mathrm{r}_{1} \mathrm{r}_{2}\right)$ 


\subsection{Somewhat Homomorphic Encryption (DGHV) [10]}

\begin{tabular}{|l|}
\hline DGHV : Somewhat homomorphic encryption \\
Encryption settings: $r$, $p$ et $q, r \sim 2^{n}, p \sim 2^{n 2}, q \sim 2^{n 5}, p$ prime, $p$ \\
is the private key. \\
$\mathrm{E}_{k}(x)=p q+2 r+x$ \\
$D_{k}(y)=(y \bmod p) \bmod 2$ \\
\hline
\end{tabular}

Proof : pq is greater than $2 r+x$

thus $y \bmod p=2 r+x$

at last $(y \bmod p) \bmod 2=(2 r+x) \bmod 2=x$

For two ciphertexts $\mathrm{c}_{1}$ et $\mathrm{c}_{2}$ :

$\mathrm{c}_{1}+\mathrm{c}_{2}=\left(\mathrm{q}_{1}+\mathrm{q}_{2}\right) \mathrm{p}+2\left(\mathrm{r}_{1}+\mathrm{r}_{2}\right)+\mathrm{m}_{1}+\mathrm{m}_{2}$

So, if : $2\left(\mathrm{r}_{1}+\mathrm{r}_{2}\right)+\mathrm{m}_{1}+\mathrm{m}_{2}<<\mathrm{p}$

Then $\left(\mathrm{c}_{1}+\mathrm{c}_{2}\right) \bmod \mathrm{p}=2\left(\mathrm{r}_{1}+\mathrm{r}_{2}\right)+\mathrm{m}_{1}+\mathrm{m}_{2}$

so, it is homomorphic for addition

$\mathrm{c}_{1} \times \mathrm{c}_{2}=\left[\mathrm{q}_{1} \mathrm{q}_{2} \mathrm{p}+\left(2 \mathrm{r}_{1}+\mathrm{m}_{1}\right)+\left(2 \mathrm{r}_{2}+\mathrm{m}_{2}\right)\right] \mathrm{p}+2\left(2 \mathrm{r}_{1} \mathrm{r}_{2}+\mathrm{r}_{1} \mathrm{~m}_{1}+\mathrm{r}_{2} \mathrm{~m}_{1}\right)+\mathrm{m}_{1} \mathrm{~m}_{2}$

So, if: $2\left(2 \mathrm{r}_{1} \mathrm{r}_{2}+\mathrm{r}_{1} \mathrm{~m}_{1}+\mathrm{r}_{2} \mathrm{~m}_{1}\right)+\mathrm{m}_{1} \mathrm{~m}_{2}<<\mathrm{p}$

Then $\left(\mathrm{c}_{1} \times \mathrm{c}_{2}\right) \bmod \mathrm{p}=2\left(2 \mathrm{r}_{1} \mathrm{r}_{2}+\mathrm{r}_{1} \mathrm{~m}_{1}+\mathrm{r}_{2} \mathrm{~m}_{1}\right)+\mathrm{m}_{1} \mathrm{~m}_{2}$

so, it is homomorphic for multiplication

\section{Simulation and Analysis}

Practical evaluation is performed in a MacBook Pro (MacBookPro 9,2), with the following characteristics:

Intel Core i5 @ $2000 \mathrm{MHz} 4 \mathrm{~GB}$ - DDR3 @ $1600 \mathrm{MHz}$

Intel HD Graphics 4000

\subsection{Result of RSA Simulation:}

For RSA, the Following Table Shows the Different Simulation Values

Table 1. Simulation Results of RSA Multiplicative Homomorphic Encryption

\begin{tabular}{|c|c|c|c|c|c|c|c|c|c|c|c|c|c|}
\hline & \multirow{3}{*}{ RSA } & \multicolumn{12}{|c|}{ Keysize } \\
\hline & & \multicolumn{3}{|c|}{256} & \multicolumn{3}{|c|}{512} & \multicolumn{3}{|c|}{1024} & \multicolumn{3}{|c|}{2048} \\
\hline & & Eirchpolien ine & $(0 \times x)$ lint & Dest(cixa) ine & Extrepplian time & 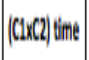 & 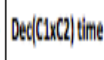 & Entrypliton tim. & $(0 \times 2)$ int & Det(cixa) line & intepplion tine & $(c x-2 x)$ in & Detc(cx) inime \\
\hline \multirow{3}{*}{$\begin{array}{l}8 \\
0 \\
-10\end{array}$} & $11 K B$ & $58 \mathrm{~ms}$ & 3Ms & $61 \mathrm{~ms}$ & 3311ms & $4 \mathrm{~ms}$ & $326 \mathrm{~ms}$ & $2384 \mathrm{~ms}$ & $11 \mathrm{~ms}$ & $2376 \mathrm{~ms}$ & 17766ms & $27 \mathrm{~ms}$ & $17668 \mathrm{~ms}$ \\
\hline & 1018 & $534 \mathrm{~ms}$ & $17 \mathrm{~ms}$ & $537 \mathrm{~ms}$ & $3284 \mathrm{~ms}$ & $29 \mathrm{~ms}$ & $3396 \mathrm{~ms}$ & $23607 \mathrm{~ms}$ & $77 \mathrm{~ms}$ & $23435 \mathrm{~ms}$ & $176848 \mathrm{~ms}$ & $234 \mathrm{~ms}$ & $176348 \mathrm{~ms}$ \\
\hline & $100 K B$ & $40607 \mathrm{~ms}$ & $976 \mathrm{~ms}$ & $40676 \mathrm{~ms}$ & $260584 \mathrm{~ms}$ & $2333 \mathrm{~ms}$ & $284736 \mathrm{~ms}$ & ..." & ..." & ...".." & ..."."' & ..."' & ....." \\
\hline
\end{tabular}




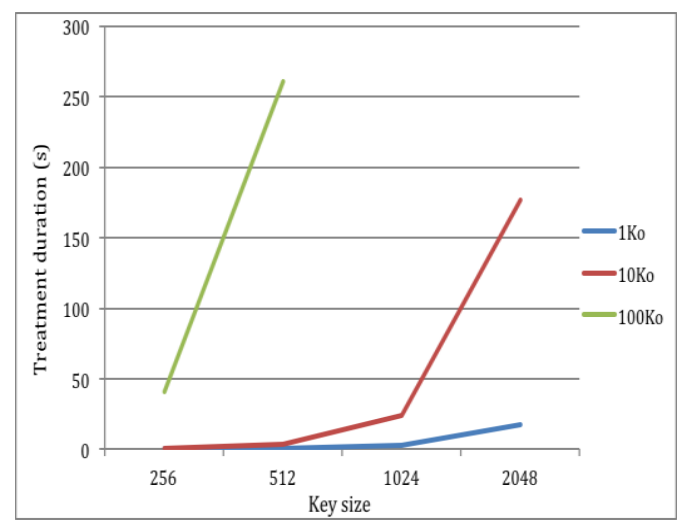

Figure 1. The RSA Encryption Time

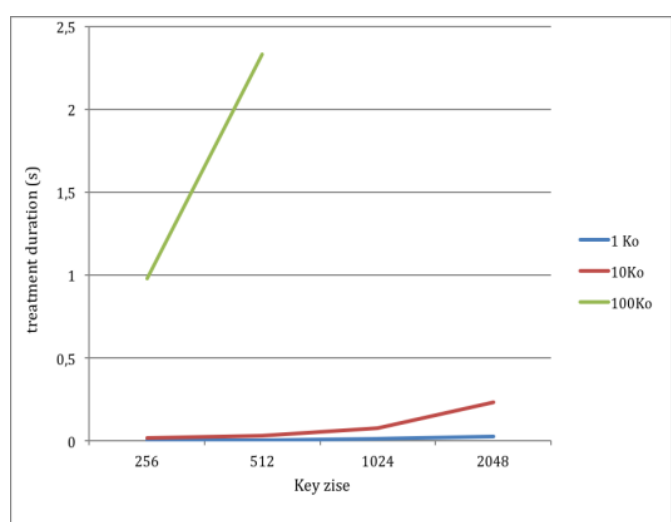

Figure 2. The Multiplication Time of Two Ciphertexts (C1xC2)

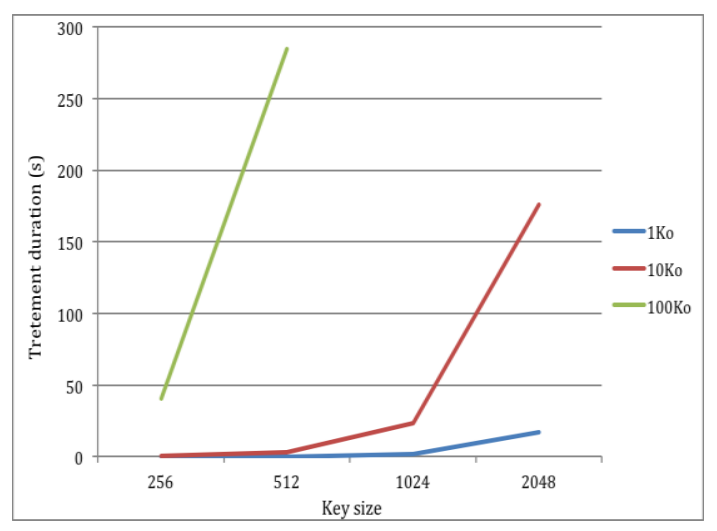

Figure 3. Decryption Time Dec (C1xC2)

- For $1 \mathrm{~KB}$ text size, RSA homomorphic cryptosystem is able to encrypt, multiply two cleartexts and decipher the result of the multiplication using a 2048 key size.

- For $10 \mathrm{~KB}$ text size, RSA homomorphic cryptosystem is able to encrypt, multiply two cleartexts and decipher the result of the multiplication using a 2048 key size, but with a very elevated processing time.

- For a 1024 key size the RSA homomorphic cryptosystem is no longer able to multiply two ciphertexts of $100 \mathrm{~KB}$ each, so it is limited in 1024 key size and 100KB cleartext size.

\subsection{Result of Paillier Simulation:}

For Paillier, The following table shows the different simulation values

Table 2. Simulation Results of Paillier Additive Homomorphic Encryption

\begin{tabular}{|c|c|c|c|c|c|c|c|c|c|c|c|c|c|}
\hline & \multirow{3}{*}{ Paillier } & \multicolumn{12}{|c|}{ Key size } \\
\hline & & \multicolumn{3}{|c|}{256} & \multicolumn{3}{|c|}{512} & \multicolumn{3}{|c|}{1024} & \multicolumn{3}{|c|}{2048} \\
\hline & & Encryption time & (CAXC) bime & $\operatorname{Dec}(\mathrm{C} \times(2))$ ime & Encryption time & (CXXC2) tim & Detec(cxa) ine & Encryption time & (CXXC) ine & $\operatorname{Det}(\mathrm{C} \times(x))$ ine & Encreption time & $(c x<2)$ ine & Det(Cx(2) int \\
\hline \multirow{3}{*}{$\begin{array}{l}8 \\
0 \\
0 \\
0\end{array}$} & $1 K B$ & $1346 \mathrm{~ms}$ & $25 \mathrm{~ms}$ & $2761 \mathrm{~ms}$ & $8671 \mathrm{~ms}$ & $71 \mathrm{~ms}$ & $17010 \mathrm{~ms}$ & $63062 \mathrm{~ms}$ & $175 \mathrm{~ms}$ & $126209 \mathrm{~ms}$ & $483979 \mathrm{~ms}$ & $526 \mathrm{~ms}$ & $971372 \mathrm{~ms}$ \\
\hline & $10 K B$ & $11438 \mathrm{~ms}$ & $214 \mathrm{~ms}$ & $21523 \mathrm{~ms}$ & $79584 \mathrm{~ms}$ & $569 \mathrm{~ms}$ & $162244 \mathrm{~ms}$ & $624020 \mathrm{~ms}$ & $1798 \mathrm{~ms}$ & $1186745 \mathrm{~ms}$ & $1395256 \mathrm{~ms}$ & $2265 \mathrm{~ms}$ & 1404403ms \\
\hline & $100 K B$ & $117612 \mathrm{~ms}$ & $2191 \mathrm{~ms}$ & $240827 \mathrm{~ms}$ & ........ & $\ldots$ & $\ldots$ & $\ldots$ & $\ldots$ & $\ldots$ & $\ldots$ & $\ldots$ & $\ldots$ \\
\hline
\end{tabular}




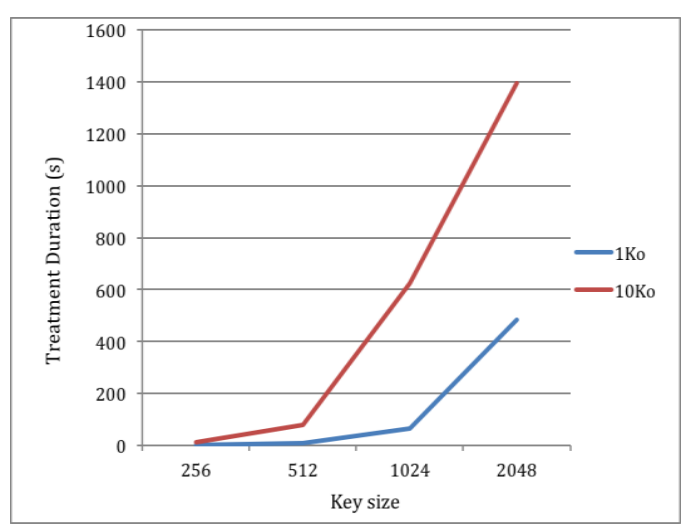

Figure 4. The Paillier Encryption Time

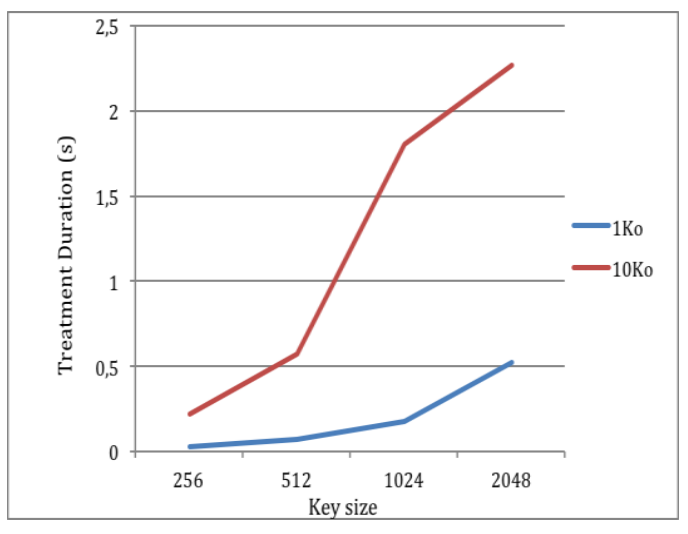

Figure 5. The Multiplication Time of Two Ciphertexts (C1xC2)

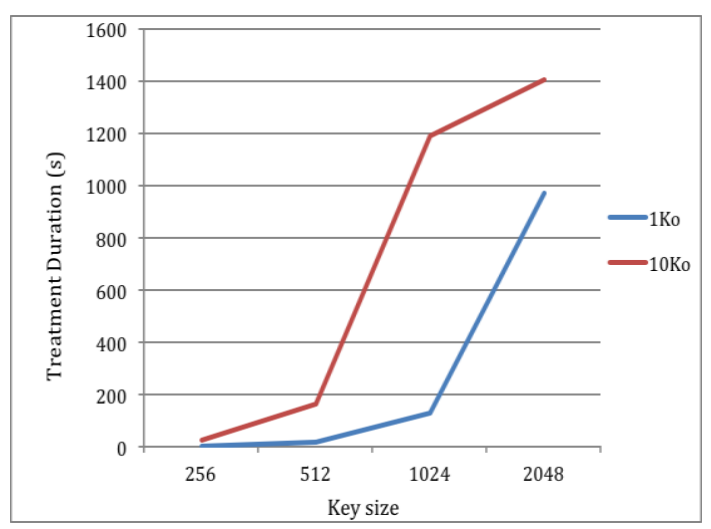

Figure 6. Decryption Time Dec (C1xC2)

- For $1 \mathrm{~KB}$ text size, Paillier homomorphic cryptosystem is able to encrypt, multiply two cleartexts and decipher the result of the multiplication using a 2048 key size, with a processing time of 16.18 minutes.

- For $10 \mathrm{~KB}$ text size, Paillier homomorphic cryptosystem is able to encrypt, multiply two cleartexts and deciphering the result of the multiplication using a 1048 key size, but with 19.77 minutes for deciphering, and 23 minutes to decipher the result using 2048 key size.

- For a 512 key size the Paillier homomorphic cryptosystem is no longer able to multiply two ciphertexts of $100 \mathrm{~KB}$ each, so it is limited at 512 key size and $100 \mathrm{~KB}$ of cleartext.

\subsection{Result of DGHV Simulation:}

For DGHV, the following tables show the different simulation values

Table 3. Simulation Results of DGHV Additive Homomorphic Encryption

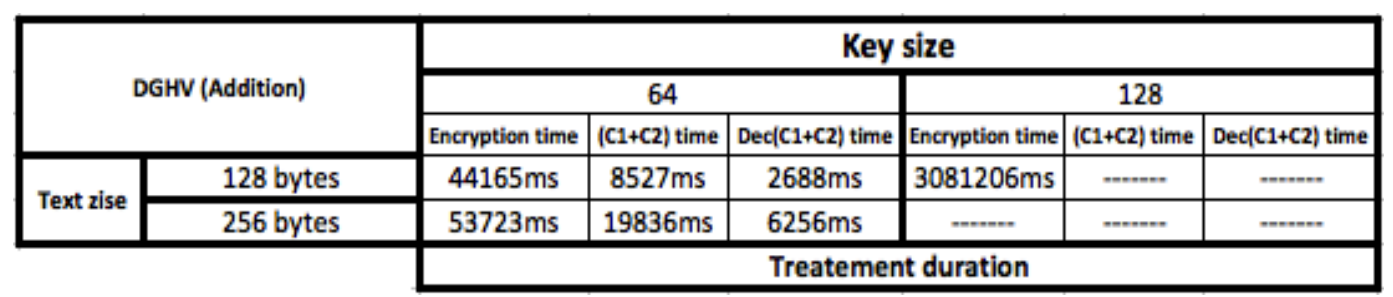


Table 4. Simulation Results of DGHV Multiplicative Homomorphic Encryption

\begin{tabular}{|c|c|c|c|c|c|c|c|}
\hline \multirow{3}{*}{\multicolumn{2}{|c|}{ DGHV (Multiplication) }} & \multicolumn{6}{|c|}{ Key size } \\
\hline & & \multicolumn{3}{|c|}{64} & \multicolumn{3}{|c|}{128} \\
\hline & & Encryption time & (C1xC2) time & Dec(C1xC2) time & Encryption time & (C1XC2) time & $\operatorname{Dec}\left(\mathrm{C}_{1 \times C 2}\right)$ time \\
\hline \multirow{2}{*}{ Text zise } & 128 bytes & $46112 \mathrm{~ms}$ & $15364 \mathrm{~ms}$ & $10401 \mathrm{~ms}$ & $2855851 \mathrm{~ms}$ & -..... & -..... \\
\hline & 256 bytes & $55254 \mathrm{~ms}$ & $\ldots$ & $\ldots$ & $-\cdots$ & $-\cdots$ & $-\cdots$ \\
\hline
\end{tabular}

- For 10KB text size, DGHV additive homomorphic cryptosystem is able to encrypt, multiply two cleartexts and deciphering the result of the multiplication using a 1048 key size, but with 19.77 minutes for deciphering, and 23 minutes to decipher the result using 2048 key size.

- For a 512 key size the DGHV multiplicative homomorphic cryptosystem is no longer able to multiply two ciphertexts of $100 \mathrm{~KB}$ each, so it is limited at 512 key size and $100 \mathrm{~KB}$ of cleartext.

\section{Hybrid Homomorphic Encryption Applied to Banking Data in the Cloud}

\subsection{Application' Scheme}

Our application scheme is as follows:

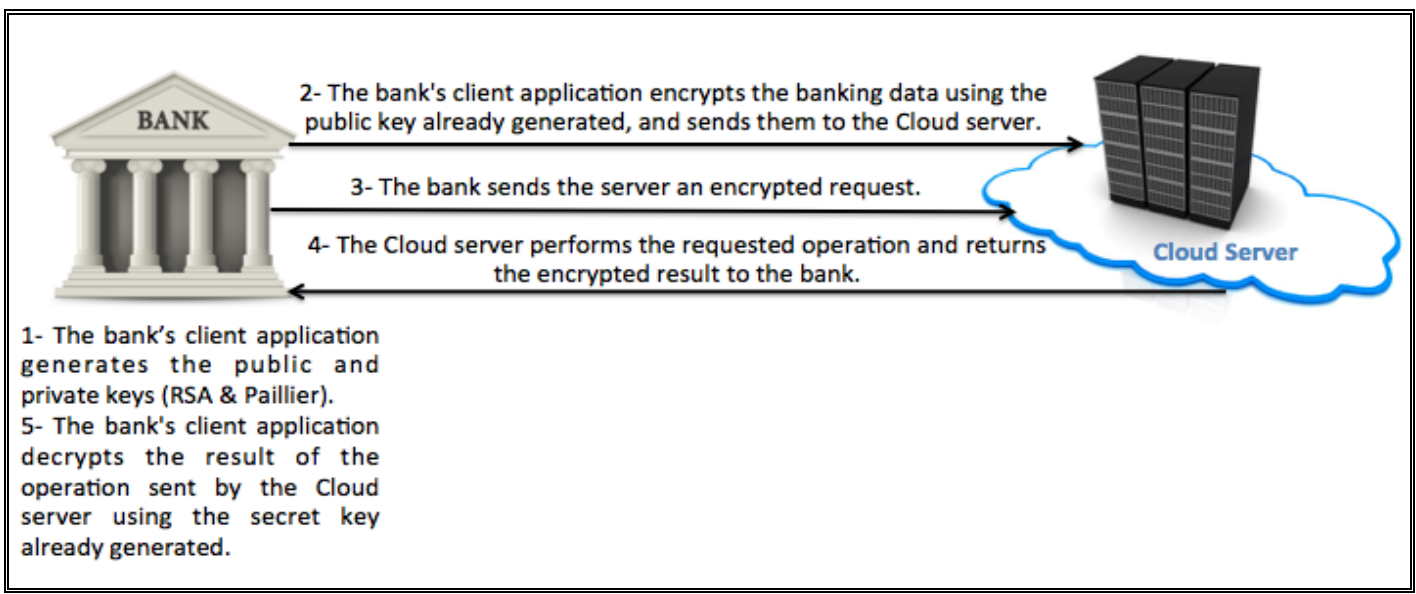

Figure 7. Scheme Representing the Link between a Client Company (Bank) and its Data Hosted in a Cloud Provider Server

VPN network linking the customer company (bank) with the server in the cloud; Our Cloud platform is set on a Citrix [11] server.

1. The bank's client application generates encryption keys with Paillier and RSA cryptosystems, encrypts data, and transmits it to the cloud server

2. The names and account numbers of customers are encrypted with RSA to enable keyword search in the encrypted data without decrypting it.

3. The sum of deposits for each customer's account is encrypted with Paillier to allow an addition operation on all the accounts of the same customer.

4. The bank sends a request to calculate the total amount of the customer's deposits

5. The Cloud server performs the calculations on encrypted data and return the encrypted result to the bank 
6. Using the private key, the client application decrypts the encrypted result and obtains the same sum as if the calculation was carried out on the raw data.

\subsection{Parameters and Results}

For our banking application, the data to be encrypted does not exceed 1KB (Name, Account number and amount of money). So we chose to execute a keyword search processing on an encrypted database and compute the sum of a customer deposits, whose data has been encrypted and stored in the cloud using a 1024 key size for RSA that requires $2,384 \mathrm{~s}$ to encrypt, $11 \mathrm{~ms}$ to calculate the product of two ciphertexts and $2,37 \mathrm{~s}$ for deciphering.

For Paillier homomorphic cryptosystem we chose a key size 512 that requires 8,671 s to encrypt, $71 \mathrm{~ms}$ to compute the sum of two ciphertexts and 17,01s for deciphering,

At the level of the bank application, we have this database:

\begin{tabular}{|c|c|c|c|c|}
\hline Account-ID & Last Name & First Name & Account Number & Amount Money \\
\hline 2 & Tebaa & Naha & 1230001 & $10000 \mathrm{NAD}$ \\
\hline 3 & Tebaa & Maha & 1230002 & $200500 \mathrm{NAD}$ \\
\hline 4 & Zkik & Karim & 1250001 & $30000 \mathrm{NAD}$ \\
\hline 5 & Rkik & Karim & 1250002 & $10500 \mathrm{NAD}$ \\
\hline 6 & El Hajji & Said & 1240001 & $20000 \mathrm{NAD}$ \\
\hline 7 & E1 Hajji & Said & 1240002 & $40000 \mathrm{NAD}$ \\
\hline
\end{tabular}

At the level of the Cloud server, data is stored encrypted:

\begin{tabular}{|c|c|c|c|c|}
\hline & 8 & 12: & $4^{\circ}$ & \\
\hline & $5:$ & 112: & $04^{\circ}$ & \\
\hline & & & & \\
\hline & & & & \\
\hline & 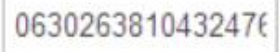 & 13. & 4. & 8 \\
\hline & $3026381043247 €$ & 07227423402023 & $15951989389425504^{*}$ & 13065914010218813318969355 \\
\hline
\end{tabular}

The customer's name encrypted using RSA

141479584167345382042993956449419
328757615144677534753157790479327
135542803873784447954116553253373
594767530870011446127375157680286
387381783207502072391425418086126
570713377529632930179550705356173
875468795690130193563599200109527
187770837622557760411789327604295
593221180808661177371167684776010
883271967759035021330748313849064
918726583987848468900000551174700
930484756624413692849669259305027
108844876761999897131417919487664
613975056267973305816332679516027
245318119774259887588570491031439

The customer account balance encrypted using Paillier

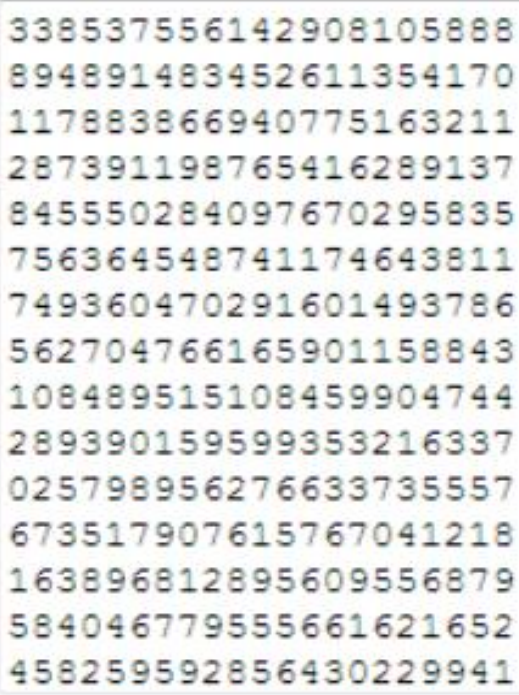



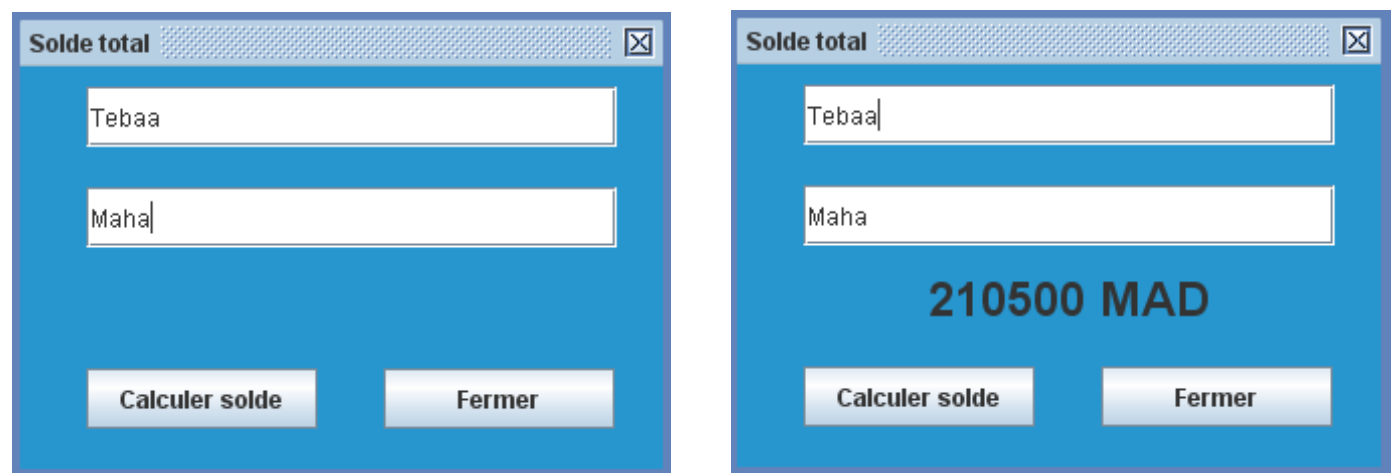

Indeed, the Balance of all the Accounts Hold by the Customer "Tebaa Maha" is 10000MAD+200500MAD $=210500 \mathrm{MAD}$

\section{Conclusion}

This hybrid system to respect the confidentiality and privacy of the database of bank accounts stored in the cloud will encourage cloud providers to integrate the homomorphic encryption in the deployment of their security solutions, to encourage banks to enjoy the benefits cloud computing [12], among the problems that exist is the management of private keys generated at the bank, you have to predict a very elevated level of protection and have a backup system to store the encryption keys and deciphering, because it is the only information that allows to access the encrypted data stored in the cloud.

\section{References}

[1] M. Tebaa and S. El Hajji, "From Single to Multi-clouds Computing Privacy and Fault Tolerance", IERI Procedia, Elsevier, vol. 10, (2014), pp. 112-118.

[2] M. Tebaa and S. El Hajji, "Secure Cloud Computing through Homomorphic Encryption", International Journal of Advancements in Computing Technology, (IJACT), vol. 5, (2013).

[3] L. Wei, H. Zhu, Z. Cao, X. Dong, W. Jia, Y. Chen and A. Vasilakos, "Security and privacy for storage and computation in cloud computing", Information Sciences, vol. 258, (2014), pp. 371-386.

[4] M. Parashar, M. Abdel Baky, I. Rodero and A. Devarakonda, "Cloud paradigms and practices for computational and data-enabled science and engineering”, Computing in Science \& Engineering, vol. 15, no. 4, (2013), pp. 10-18.

[5] S. Pearson, Y. Shen and M. Mowbray, "A privacy manager for cloud computing”, Cloud Computing, Springer Berlin Heidelberg, (2009), pp. 90-106.

[6] P. Manish, A. Moustafa, R. Ivan and D. Aditya, "Cloud paradigms and practices for computational and data-enabled science and engineering", Computing in Science \& Engineering, IEEE, vol. 15, no 4, (2013), pp. 10-18.

[7] AWS: http://www.cloudmagazine.fr/avis-expert/je-construis-mon-datacenter-dans-le-cloud-en-5minutes -top-chrono.

[8] R. Rivest, A. Shamir and L. Adleman, "A method for obtaining digital signatures and public key cryptosystems”. Communications of the ACM, 120-126, 1978. Computer Science, Springer, (1999), pp. 223-238.

[9] P. Paillier. "Public-key cryptosystems based on composite degree residuosity classes", 18th Annual Eurocrypt Conference (EUROCRYPT'99), Prague, Czech Republic, vol. 1592, (1999).

[10] M. Van Dijk, C. Gentry, S. Halevi and V. Vaikuntanathan, "Fully homomorphic encryption over the integers", Advances in cryptology- EUROCRYPT 2010, Lecture Notes in Computer Science, Springer, vol. 6110, (2010), pp. 24-43.

[11] R. van der Linden, D. Halls, S. Waterhouse and P. Benoit, "Systems and methods for establishing a cloud bridge between virtual storage resources U.S. Patent No. 8,578,076", Washington, DC: U.S. Patent and Trademark Office, (2013).

[12] D. Chen and H. Zhao, "Data security and privacy protection issues in cloud computing", Computer Science and Electronics Engineering (ICCSEE), 2012 International Conference, IEEE, vol. 1, (2012), pp. 647-651. 
International Journal of Security and Its Applications Vol. 9, No. 6 (2015) 